\title{
RELAÇÃO ENTRE DEPENDÊNCIA DE INTERNET E TRAÇOS DE PERSONALIDADE EM ESTUDANTES UNIVERSITÁRIOS
}

Marina Capellão Becker

Psicóloga. Universidade do Vale do Taquari UNIVATES, Brasil.

\section{Raquel de Melo Boff}

Doutora em Psicologia Clínica pela PUCRS. Docente do curso de Psicologia na Universidade do Vale do Taquari - UNIVATES, Brasil.

\section{Ana Luísa Freitag}

Universidade do Vale do Taquari - UNIVATES. Ciências Biológicas e da Saúde, Brasil.
RESUMO: Objetivou-se avaliar a associação entre os traços de personalidade e uso excessivo de internet em universitários. Este estudo transversal, quantitativo, realizado em 2017 com 259 universitários de uma instituição de ensino superior particular, na região Sul do Brasil. Utilizou-se um instrumento sociodemográfico, a escala de Personalidade de Comrey (CPS) e internet Addiction Test (IAT). Os dados foram analisados no Statistical Package for Social Sciences, versão 23.0, por meio de testes estatísticos Kolmogorov Smirnov, Spearman, $t$ de student, Regressão Logística Multivariada e o método Bakcward ( $p \leq 0,05)$. A maioria dos participantes usufrui da internet em casa, através do celular, para fins de utilização de redes sociais e com prevalência do sexo feminino. Existe correlação negativa bem fraca entre a escala T do CPS e a IAT $(p=0,144)$, e correlação negativa fraca entre as escalas A $(p=0,262)$ e $S(p=0,231)$. Sugeremse mais estudos e intervenção neste campo e com este público-alvo.

PALAVRAS-CHAVE: Internet; Estudantes; Ensino superior.

\section{RELATIONSHIP BET WEEN INTERNET DEPENDENCY AND PERSONALITY TRAITS IN UNDERGRADUATES}

\begin{abstract}
The association between personality traits and excessive usage of the Internet in undergraduates is investigated through a transversal and quantitative study in 2017 with 259 undergraduates in a private higher institution in south Brazil. The sociodemographic tool, Comprey's Personality Scale and the Internet Addiction Test were employed. Data were analyzed with Statistical Package for Social Sciences 23.0, by statistic tests Kolmogorov Smirnov, Spearman, Student's t, Multivariate Logistic Regression and by Bakcward method $(p \leq 0.05)$. Most participants, particularly females, use the Internet at home, through their mobiles, in social networks. There is negative co-relationship between scale T of CPS and IAT $(p=0.144)$, and a weak negative co-relationship between scales A $(p=0.262)$ and $S$ $(p=0.231)$. More studies and field intervention are required in this field and target population.
\end{abstract}

KEY WORDS: Internet; Students; Education Higher.

\section{INTRODUÇÃO}

Autor correspondente

Ana Luísa Freitag

analuisafreitag@gmail.com
A utilização da internet e redes sociais tem crescido consideravelmente em nível global nas últimas décadas. O Brasil é o terceiro país no ranking mundial de usuários de internet e primeiro em período de tempo de acesso à ferramenta ${ }^{1}$. Indubitavelmente, a 
internet uniu as pessoas, os grupos e as comunidades que possuem interesses em comum. Porém, junto a este processo se observa a tendência de indivíduos à sua utilização excessiva, o que vem sendo estudado como possível transtorno psicológico, principalmente no que diz respeito às redes sociais e jogos eletrônicos ${ }^{2,3}$. Embora não haja consenso na literatura em relação à terminologia mais apropriada para a condição, optouse por utilizar o termo "dependência de internet", pois esta é a nomenclatura predominantemente utilizada em pesquisas recentes ${ }^{4,5,6,7}$.

Em um estudo com universitários, o comprometimento funcional é apontado como um importante critério diagnóstico para dependência de internet $^{8}$. Ainda, o número de horas que alguém fica na internet não é uma variável confiável para estabelecer relação de dependência, mas o impacto do uso da internet sobre a vida do indivíduo - prejuízo social, financeiro, afetivo e profissional advindo deste uso. Além disto, destaca-se a forma como o indivíduo lida com estes prejuízos que também devem ser material de observação?.

Quanto à população de risco, jovens e adolescentes vêm se destacando, uma vez que se encontram expostos à utilização da internet do que qualquer outro grupo etário ${ }^{10}$. Também, um estudo com jovens adultos iranianos indicou diferenças sensíveis referente ao gênero, sendo o risco de desenvolvimento de dependência maior para homens do que para mulheres 5 .

Sabe-se mais em relação às consequências que a dependência de internet traz do que sobre as possíveis causas ou variáveis que contribuem para a vulnerabilidade do indivíduo. Por se tratar de uma atividade comum ao cotidiano da maior parte das pessoas, considera-se necessário identificar variáveis que contribuam para a vulnerabilidade do indivíduo ao invés de partir do pressuposto de que qualquer pessoa poderia perder o controle e se tornar dependente de internet ${ }^{11}$.

Ainda que tenha ocorrido tentativas de descrição da personalidade de risco para a dependência de internet, na literatura não se chegou a um consenso quanto aos traços mais predominantes. Um estudo realizado em Portugal coloca que o perfil do dependente de internet pode contemplar algumas das seguintes dimensões: depressão, distúrbio bipolar, compulsão sexual, hostilidade, transtorno obsessivo-compulsivo (TOC) e solidão. ${ }^{12}$ Outro estudo com universitários da Turquia encontrou maiores taxas de tabagismo e alcoolismo entre dependentes de internet, o que remete à ideia de estratégia de manejo de dificuldades ${ }^{13}$.

Uma hipótese levantada seria de que as pessoas dependentes e com algum transtorno mental usem a internet para alívio de sintomas e, assim, se apresentam enquanto população vulnerável para a dependência de internet. Ao enfrentar dificuldades nas relações "reais", uma forma de fuga seria recorrer à internet. Ao tentar compensar a falta de habilidade social, a depressão, a baixa autoestima e a solidão, o uso se torna extremamente reforçador, aumentando o risco de ser dependente de internet ${ }^{14}$.

A partir do exposto, observa-se a necessidade de mais estudos na área de dependência de internet no Brasil, a fim de estabelecer etiologia e promover formas de prevenção e tratamento para a condição. Para tanto, no presente estudo objetivou-se avaliar a associação entre traços de personalidade e uso excessivo de internet em estudantes universitários. Pela possível relação entre estas variáveis, buscou-se analisar quais traços são potenciais preditivos do uso excessivo desta ferramenta.

\section{METODOLOGIA}

\section{AMOSTRA}

Participaram do estudo transversal e quantitativo 259 universitários, de 18 a 60 anos, regularmente matriculados em uma Instituição de Ensino Superior (IES) privada da região Sul do Brasil, em um dos seguintes cursos: administração; biomedicina; ciências contábeis; comércio exterior; direito; engenharia da computação; engenharia de produção; engenharia de software; estética e cosmética; fisioterapia; gestão de recursos humanos; gestão de micro e pequenas empresas; gestão financeira; jornalismo; psicologia; publicidade e propaganda; redes de computadores e/ou sistemas de informação. A composição da amostra foi feita por conveniência, não excluindo nenhum participante. 


\section{PROCEDIMENTOS}

A seleção das turmas foi realizada juntamente com os centros de curso, a fim de abranger os mais diferentes cursos e áreas de estudo. A coleta dos dados ocorreu nas salas de aula dos estudantes durante os meses de maio e abril de 2017 , mediante a autorização prévia dos professores. A aplicação dos instrumentos teve duração de 40 minutos e média de 40 estudantes em cada turma. Após a leitura/entendimento e assinatura do Termo de Consentimento Livre e Esclarecido (TCLE) por parte dos estudantes, foram entregues os instrumentos autorrespondidos.

\section{INSTRUMENTOS}

Ficha de dados sociodemográficos: instrumento criado pelos pesquisadores, solicitando dados referentes à idade, ao sexo, ao curso e aos comportamentos relacionados à utilização da internet.

Internet Addiction Test (IAT): primeiro instrumento validado para a avaliação da dependência de internet, também validada para a língua portuguesa $(\alpha=0.85) .{ }^{15}$ Trata-se de uma medida fidedigna que classifica os indivíduos em dependentes e não dependentes e específica gravidade sintomatológica. É composto de 20 questões em que o indivíduo pontua por meio de uma escala Likert de cinco pontos entre "Não aplicável", "Raramente", "Ocasionalmente", "Frequentemente", "Geralmente" e "Sempre".

Escala de personalidade de Comrey (CPS): validado no Brasil, o CPS é de uso restrito aos psicólogos que avalia oito dimensões da personalidade: confiança vs. atitude defensiva (escala T, $\alpha=0.589$ ); ordem vs. falta de compulsão (escala $\mathrm{O}, \alpha=0,604$ ); conformidade social vs. rebeldia (escala $C, \alpha=0.327$ ); atividade vs. falta de energia (escala A, $\alpha=0,685$ ); estabilidade emocional vs. instabilidade (escala $S, \quad \alpha=0.755$ ); extroversão vs. introversão (escala $\mathrm{E}, \alpha=0.839$ ); masculinidade vs. feminilidade (escala $\mathrm{M}, \alpha=0.614$ ); empatia vs. egocentrismo (escala $\mathrm{P}, \alpha=0.735$ ). A escala mede ainda validade (escala $\mathrm{V}, \alpha=0.397$ ) e tendenciosidade nas respostas (escala $\mathrm{R}, \alpha=0.708) .{ }^{16} \mathrm{Cada}$ dimensão possui duas polaridades, assim quanto mais próximo de zero, mais equilíbrio há em relação a esta dimensão. Por exemplo, no caso da escala $\mathrm{E}$, quanto mais distante de zero para cima, mais extrovertido; quanto mais longe de zero para baixo, mais introvertido.

\section{ANÁLISE DOS DADOS}

A análise de dados foi realizada por meio de estatística descritiva e multivariada, utilizando-se para tratamento estatístico de dados o Statistical Package for Social Sciences (SPSS), versão 23.0. Os dados descritivos foram analisados por meio de frequências e tendências centrais. A normalidade dos dados foi avaliada por meio do teste Kolmogorov Smirnov, adotando a significância de $95 \%$. Foram empregadas análises de correlação de Spearman para dados não paramétricos. Foram utilizados testes $t$ de student para amostras independentes a fim de analisar as diferenças entre as médias com base no sexo do participante. Uma regressão logística multivariada foi aplicada para avaliar potenciais preditivos sobre a variável dependente escore total no IAT. O método Bakcward foi aplicado e como variáveis independentes foram incluídos os escores relativos as oito escalas de personalidade do CPS.

\section{ASPECTOS ÉTICOS}

A pesquisa teve aprovação do Comitê de Ética em Pesquisa, sob o número do parecer 1.895.711, respeitando os aspectos éticos conforme as diretrizes e normas nacionais e internacionais de pesquisas que envolvem os seres humanos.

\section{RESULTADOS}

Os participantes foram 114 (44\%) do sexo masculino e 145 (56\%) do sexo feminino, com média de idade de 22,74 ( $D P=5.48)$. Dados da amostra referentes à etnia, ao estado civil, à situação ocupacional, à renda familiar e às áreas de estudos seguem na Tabela 1. Informações relativas aos cursos de graduação aos quais pertencem os estudantes seguem na Tabela 2. 
Tabela 1. Caracterização sociodemográfica dos estudantes universitários $(\mathrm{n}=259)$

\begin{tabular}{lcc}
\hline Variáveis & N & $\%$ \\
\hline Etnia & & \\
\hline Brancos & 252 & 97.3 \\
Afrodescendentes & 7 & 2.7 \\
\hline Estado civil & 227 & 87.6 \\
\hline Solteiro & 32 & 12.4 \\
$\quad$ Casado & & \\
\hline Situação ocupacional & 201 & 77.6 \\
\hline Ativo/trabalhando & 54 & 20.8 \\
$\quad$ Desempregado/afastado & & \\
\hline Renda familiar & 89 & 34.4 \\
\hline 1 a 3 salários mínimos & 101 & 39 \\
3 a 5 salários mínimos & 69 & 26.6 \\
$\quad$ Mais de 5 salários mínimos & & \\
\hline Área de estudo & 136 & 25.5 \\
\hline Ciências exatas & 81 & 31.3 \\
Ciências biológicas & 42 & 16.2 \\
Ciências humanas & &
\end{tabular}

Tabela 2. Relação dos cursos de graduação dos participantes

\begin{tabular}{lcc}
\hline \multicolumn{1}{c}{ Cursos } & N & \% \\
\hline Administração & 23 & 8.9 \\
Biomedicina & 25 & 9.7 \\
Comércio exterior & 5 & 1.9 \\
Ciências contábeis & 45 & 17.4 \\
Direito & 22 & 8.5 \\
Engenharia da computação & 5 & 1.9 \\
Engenharia de software & 6 & 2.3 \\
Estética e cosmética & 22 & 8.5 \\
Fisioterapia & 3 & 1.2 \\
Gestão financeira & 6 & 2.3 \\
Gestão de micro e pequenas empresas & 24 & 9.3 \\
Gestão de recursos humanos & 5 & 1.9 \\
Jornalismo & 8 & 3.1 \\
Psicologia & 31 & 12 \\
Publicidade e propaganda & 9 & 3.5 \\
Redes de computadores & 5 & 1.9 \\
Sistemas de informação & 14 & 5.4 \\
\hline
\end{tabular}

Fonte: Dados da pesquisa (2019).

Em relação ao local de maior utilização da internet pelos participantes, $66 \%$ utilizam em casa, $20.5 \%$ no trabalho e $13.5 \%$ na universidade e outros lugares. A maioria dos estudantes (67.2\%) utiliza a internet principalmente através do celular; outros $26.6 \%$ acessam principalmente através do computador e os demais (13.4\%) através de tablets e outras ferramentas.
Um importante dado coletado diz respeito ao motivo pelo qual a maioria dos estudantes acessa a internet, sendo este para fins de utilização de redes sociais (52.1\%). Uma porcentagem de 35.2\% acessa por motivos de estudo ou trabalho, e os demais (jogos, compras e outros) correspondem a $12.7 \%$.

Mais da metade dos universitários pesquisados (51\%) declara já ter deixado de realizar alguma tarefa importante para poder ficar conectado. Também, a maioria (52.9\%) dos entrevistados refere que pessoas próximas/ familiares já reclamaram do tempo que permanecem conectados à internet; mencionam sentir dificuldades em resistir ao impulso ou tentação de conectar-se 41.3\% e $49 \%$ entendem que a vida seria vazia/chata/sem graça caso não tivessem acesso à internet.

Em relação à maneira como se sentem quando não utilizam a internet, $16.2 \%$ relata sentir-se deprimido e $13.9 \%$ se sente nervoso/ansioso; declaram algum diagnóstico relativo à saúde mental $25.9 \%$ dos respondentes. Destes, $24.7 \%$ obtiveram diagnóstico de depressão e ansiedade, $1.2 \%$ diagnóstico de TOC e $0.4 \%$ diagnóstico de dependência química.

Conforme Tabela 3, existe correlação negativa bem fraca entre a escala $\mathrm{T}$ (confiança $\mathrm{X}$ atitude defensiva) do CPS e a dependência de internet (internet Addiction Test - IAT $)(p=0,144)$. Há também correlação negativa significativa e fraca entre a escala A (atividade $\mathrm{X}$ falta de energia) deste instrumento e a dependência de internet $(p=0,262)$, o que permite compreender que quanto maior a atividade, menor a possibilidade de desenvolvimento de dependência. Encontrou-se além desses correlação negativa fraca entre a escala $\mathrm{S}$ (estabilidade $\mathrm{X}$ instabilidade emocional), o que demonstra que quanto mais estável, menos probabilidade de desenvolvimento de dependência. 
Tabela 3. Descritivas e correlação entre escalas do CPS e o escore total do IAT

\begin{tabular}{cccc}
\hline Variáveis & Média $(\mathbf{D P})$ & $\mathbf{p}($ Spearman $)$ & $\mathbf{P}$ \\
\hline IAT & $24,66(12,41)$ & & - \\
Escala T & $38,03(5,42)$ & $-0,144^{*}$ & 0,021 \\
Escala O & $51,23(6,33)$ & $-0,289$ & $<0,001$ \\
Escala C & $38,97(5,43)$ & $-0,009$ & 0,883 \\
Escala A & $47,57(8,26)$ & $-0,262^{* *}$ & $<0,001$ \\
Escala S & $46,51(8,38)$ & 0,231 & $<0,001$ \\
Escala E & $45,14(9,25)$ & $-0,106$ & 0,089 \\
Escala M & $36,17(10,08)$ & 0,0086 & 0,166 \\
Escala P & $45,03(6,82)$ & $-0,168$ & 0,007 \\
Escala V & $13,08(5,780$ & 0,026 & 0,678 \\
Escala R & $39,55(8,09)$ & $-0,190^{* *}$ & $<0,001$ \\
\hline
\end{tabular}

A*correlação é significativa ao ponto de 0,05 (bilateral); ${ }^{* * A}$ correlação é significativa ao ponto de 0,01 (bilateral).

Fonte: Dados da pesquisa (2019)

A partir de análise por meio do teste $t$ de student para amostras independentes, encontrou-se significativa diferença entre estudantes do sexo feminino e sexo masculino no que tange ao uso da internet $(\mathrm{F}=0,397$; $\mathrm{t}=2,207 ; \mathrm{p}=0,028$ ), considerando que estudantes do sexo masculino tem escore maior nessa variável. Ainda, ao comparar traços de personalidade entre grupos por sexo, há diferença significativa demonstrando maior atividade no sexo feminino na escala A $(\mathrm{F}=0,308 ; \mathrm{t}=2,798$; $\mathrm{p}<0,001)$; maior estabilidade emocional através da escala $S$ no sexo masculino $(F=1,166 ; t=5,688 ; p<0,001)$; maiores níveis de empatia e altruísmo por meio da escala $\mathrm{P}$ no sexo feminino $(\mathrm{F}=1,908 ; \mathrm{t}=-6,028 ; \mathrm{p}<0,001)$.
A Tabela 4 apresenta os resultados referentes ao modelo de regressão logística apontado para explicar possíveis preditores de uso da internet. De acordo com os resultados o modelo final foi elencado em quatro etapas (step), sendo que aceitos os cumprimentos dos pressupostos básicos para a técnica, a estimativa para o valor $\mathrm{R}^{2}$ foi de 0.191 , muito próximo do $\mathrm{R}^{2}$ ajustado $(0,168)$, que permite inferir que os dois testes preditores influenciaram 19,1\% da variação da pontuação total do IAT.

\section{DISCUSSÃO}

Poucos estudos internacionais abordam a temática pesquisada. Além desta limitação, no presente estudo utilizou-se a escala de personalidade de Comrey para mensurar a variável traço de personalidade e encontraram-se, posteriormente, dificuldades em relacionar a outros estudos. As pesquisas encontradas com dados significativos utilizaram escalas baseadas no Modelo dos Cinco Grandes Fatores da Personalidade, também conhecido como Big Five, desenvolvido por McDougall na década de 30, que mais tarde inspirou os estudos de Fiske (1949), Borgatta (1964), e Tupes e Christal (1992). ${ }^{17}$

Porém, uma aproximação foi possível com um estudo realizado com participantes da Polônia, Turquia e Ucrânia, utilizando como base para mensuração o Big Five, onde se encontrou correlação entre dependência de internet e instabilidade emocional $^{18}$.

Tabela 4. Estimativas para a análise de regressão linear múltipla para responder pelo uso de internet

\begin{tabular}{ccccc}
\hline Variáveis independentes & \multicolumn{2}{c}{ Coeficiente de regressão } & \multicolumn{2}{c}{$\begin{array}{c}\text { Coeficiente } \\
\text { padronizado }\end{array}$} \\
\cline { 2 - 4 } Sig. \\
ESCORE DO IAT* & $\boldsymbol{\beta}$ & Erro padrão b & $\boldsymbol{\beta}$ \\
\hline (Constante) & & & & \\
Escala T: confiança X atitude defensiva & 90,446 & 9,784 & $-0,129$ & 0,000 \\
Escala O: ordem X falta de compulsão & $-0,295$ & 0,132 & $-0,207$ & 0,001 \\
Escala A: atividade X falta de energia & $-0,405$ & 0,119 & $-0,189$ & 0,002 \\
Escala E: extroversão X introversão & $-0,284$ & 0,092 & $-0,131$ & 0,032 \\
Escala P: empatia X egocentrismo & $-0,175$ & 0,081 & $-0,100$ & 0,092 \\
Escala V: validade & $-0,182$ & 0,108 & 0,110 & 0,071 \\
Escala R: Tendenciosidade de resposta & 0,235 & 0,130 & $-0,120$ \\
\hline
\end{tabular}

*; Método Bacward conditional; $\mathrm{R}^{2}=0,191 / \mathrm{R}^{2}$ ajustado=0,186/Anova (Fcalc-8,441; $\mathrm{p}=0,000$ ). Durbin Whatson $=1,938$ Fonte: Dados da pesquisa (2019). 
No presente estudo encontrou-se correlação negativa fraca entre estabilidade e dependência, o que corrobora com o estudo citado.

Pesquisas anteriores apresentam que as comorbidades psiquiátricas que mais comumente ocorrem junto com a dependência de internet são os transtornos do humor, transtorno de ansiedade, transtorno de déficit de atenção e hiperatividade e transtorno de uso de substâncias ${ }^{919}$. Por este motivo, hipotetizou-se anteriormente que a instabilidade emocional pudesse estar associada à dependência de internet, o que foi confirmado.

Outra hipótese dizia respeito ao traço de personalidade falta de energia. Pelo fato de a depressão ter sido associada ao comportamento dependente de internet, formulou-se esta hipótese de associação. ${ }^{19}$ Isto, porque, fazem parte deste construto: inatividade física, desmotivação e falta de vigor e energia, que são considerados sintomas deprimidos pelo Manual de Diagnóstico e Estatístico de Transtornos Mentais 5 $\left(\right.$ DSM 5) ${ }^{20}$. A correlação encontrada foi negativa e fraca, dado que pode ser interpretado como quanto maior a atividade, menor a tendência à dependência. Apesar de fraca, a correlação é existente, o que confirma a hipótese.

Pelos dados encontrados em literatura que descrevemcaracterísticasassociadasàdependência(humor deprimido, impulsividade, busca por sensações, baixa autoestima, timidez e capacidade de atenção reduzida), formulou-se a hipótese (atualmente confirmada) de que a atitude defensiva também pudesse estar associada. Isto, porque define indivíduos desconfiados, retraídos e que possuem opinião inicialmente negativa em relação aos outros 9 . Para esta escala encontrou-se correlação negativa bem fraca, porém existente. Isto significa de forma sensível que quanto maior a confiança menor seria a tendência ao desenvolvimento da dependência, confirmando a hipótese.

A literatura aponta que os usuários dependentes não utilizam tanto a internet para acessar e-mails e navegar em sites, mas buscam facilitar suas relações afetivas por meio da comunicação interpessoal simultânea, ou seja, para conhecer pessoas e obter experiências sociais de forma online ${ }^{21,22}$. Quanto a isto, um estudo realizado na Itália, em 2017, correlacionou traços de personalidade, dependência de internet e vício no Facebook, e encontrou correlação positiva entre estas duas últimas variáveis. ${ }^{18}$ No presente estudo, mais da metade (52.1\%) dos participantes assinalou que o principal motivo de acesso seria pelas redes sociais, um dado significativo. Porém, destes, nem todos são dependentes, o que impossibilita a correlação com dependência de internet.

Juntamente com o estudo, citado anteriormente, que encontrou dados que apontam para diferenças entre gênero quanto ao desenvolvimento da dependência, o presente estudo verificou diferença estatisticamente significativa quanto a esta questão5. Estudantes do sexo masculino teriam maior tendência ao desenvolvimento de dependência. Além disso, independente dos dados relacionados ao IAT, alguns dados relativos às escalas de personalidade são totalmente novos como, por exemplo: relação entre sexo feminino e atividade, estabilidade emocional relacionada ao sexo masculino, e maiores níveis de empatia por parte do sexo feminino.

Outra tendência encontrada foi a de que há relação negativa quanto às escalas $\mathrm{T}, \mathrm{O}, \mathrm{A}$ e $\mathrm{E}$ com a dependência, o que significa que a atitude defensiva, a falta de compulsão, a falta de energia e a introversão correspondem a 19,1\% de variação da dependência em universitários. Em conjunto, tais escalas explicam tendência ao desenvolvimento de dependência. Não foram encontrados estudos anteriores que tenham verificado dados semelhantes, o que impossibilita discussão.

No DSM 5, a dependência de internet não é mencionada, sendo que o apêndice "Condições para estudos posteriores" abrange apenas "Transtorno do Jogo pela Internet", compondo uma categoria diagnóstica focada apenas no jogo pela internet e não na dependência de internet em $\mathrm{si}^{20}$. Nesse sentido, vê-se a importância do aperfeiçoamento de um método padrão de identificação e estruturação de diagnóstico a fim de validar esta dependência como uma categoria diagnóstica para elaborar formas de prevenção e tratamento. 


\section{CONCLUSÃO}

O modo de vida das pessoas e a dinâmica social vêm se modificando ao longo dos últimos anos, assim como a forma que os indivíduos se relacionam com a internet nos diferentes contextos. São indiscutíveis os inúmeros benefícios da utilização da internet, no entanto, o uso excessivo pode trazer prejuízos comportamentais, sociais e de saúde.

O estudo apontou que mais da metade dos participantes usufruem da internet em casa, através do celular, para fins de utilização de redes sociais e com prevalência do sexo feminino. Ademais, a maioria dos universitários declara que já deixou de realizar alguma tarefa importante para ficar conectado e que pessoas próximas/familiares já reclamaram do tempo que permanece conectados. Em relação aos traços de personalidade, a pesquisa mostrou que existe uma correlação negativa bem fraca entre a escala T do CPS e a dependência de internet e uma correlação negativa significativa e fraca entre a escalas A e $S$ e a dependência de internet.

Quanto às limitações encontradas ao longo deste estudo, considerando a utilização do CPS e não de algum questionário baseado no Big Five, houve impossibilidade de comparação com dados encontrados por estudos anteriores. Desta forma, a generalização dos dados deve ser cautelosa.

Apesar disso, entende-se ser importante a análise e comparação de diferentes questionários de traços de personalidade com a dependência de internet a fim de abranger um maior número de possíveis traços relacionados à esta variável. Observa-se que na literatura são relacionadas estas duas variáveis de maneira empobrecida pelo fato de utilizar apenas um tipo de mensuração. Desta forma, sugere-se que não esgotem os estudos acerca da relação entre dependência de internet e os traços de personalidade.

\section{REFERÊNCIAS}

1. Azevedo JC, Souza CHMS, Nascimento G. Ciberdependência: o papel das emoções na dependência de tecnologias digitais. Texto Livre. 2014 [acesso em 2017 maio 17]7(2):148-161. Disponível em: http://www.periodicos.letras.ufmg. br/index.php/textolivre/article/view/6173. DOI: https://doi.org/10.17851/1983-3652.7.2.148-161.

2. Lima NL, Moreira JO, Stengel M, Maia LM. As redes sociais virtuais e a dinâmica da internet. Gerais: Revista Intersinstitucional de Psicologia. 2016;9(1):90-109.

3. Oliveira F, Pasqualini KC. Os dependentes de internet no Brasil: realidade ou mito entre os universitários. Mimesis Bauru. 2014:35(1), 95-140.

4. Yang, X.; Zhu, L.; Chen, Q.; Song, P.; Wang, Z. Parent marital conflict and Internet addiction among Chinese college students: The mediating role of father-child, mother-child, and peer attachment. Computers in Human Behavior. [internet] 2016 [acessso em 2018 jul 20] 59:221229. Disponível em: https://www.sciencedirect.com/ science/article/pii/S0747563216300504?via\%3Dihub. DOI: https://doi.org/10.1016/j.chb.2016.01.041.

5. Ostovar S, Allahyar N, Aminpoor H, Moafian F, Nor MBM; Griffiths MD. Internet Addiction and its Psychosocial Risks (Depression, Anxiety, Stress and Loneliness) among Iranian Adolescents and Young Adults: A Structural Equation Model in a CrossSectional Study. International Journal of Mental Health and Addiction. 2016; 14:257-67.

6. Li D, Zhang W, LI X, Zhou Y, Zhao L, Wang Y. Stressful life events and adolescent Internet addiction: The mediating role of psychological needs satisfaction and the moderating role of coping style.Computers in Human Behavior. [internet] 2016 [acesso em 2017 abr 14] 63:40815.Disponível em:https://www.sciencedirect.com/ science/article/pii/S0747563216303971?via\%3Dihub. DOI: https://doi.org/10.1016/j.chb.2016.05.070.

7. Wu X, Zhang Z, Zhao F, Wang W, Li Y, BiL, et al. Prevalence of Internet addiction and its association with social support and other related factors among adolescents in China. Journal of Adolescence. 2016; 


\section{3:103-111.}

8. Ko CH, Liu GC, Hsiao S, Yen J Y, Yang MJ, Lin WC, et al. Brain activities associated with gaming urge of online gaming addiction. J Psychiatr Res.[internet] 2009 [acesso em 2017 abr 28]43(7):739-747. Disponível em: https:// www.sciencedirect.com/science/article/pii/ S002239560800229X?via\%3Dihub. DOI: https://doi. org/10.1016/j.jpsychires.2008.09.012.

9. Young KS. Compulsive Surfing. Net addiction. [internet] Estados Unidos; 2012 [acesso em 2017 jun. 12]. Disponível em: http://netaddiction.com/?option $=\mathrm{com}_{-}$ content\&view $=$ article\&id $=62 \% 20 \& I t \_$emid $=85$.

10. Silva RA, Silva PMF, Pereira JFM, Santos DCD, Raposo JCS, Gomes BMR. Adolescentes e abuso de tecnologias: um indicativo de problemas comportamentais? Adolescência e Saúde. 2017;14(3):77-82.

11. Li W, O'brien JE, Snyder SM, Howard MO. Characteristics of internet addiction/pathological internet use in U.S. university students: a qualitative-method investigation.PLoSOne. [internet] 2015 [acesso em 2017 abr 17]. Disponível em:https:/journals.plos.org/plosone/ article?id =10.1371/journal.pone.0117372. DOI: https://doi.org/10.1371/journal.pone.0117372

12. Pontes HOM. A Dependência à Internet: Fundamentação empírica, teórica e clínica - da psicologia e psicometria à ciber-psicologia [dissertação]. Portugal: Instituto Universitário Ciências Psicológicas Sociais e da Vida; 2013. 201 p.

13. Alaçam H, Atesçi FÇ, Şengül AC, Tümkaya S. The relationship between smoking \& alcohol use and internet addiction among the university students. AnadoluPsikiyatriDerg. [internet] 2015 [acesso em 2017 mai 02]16(6):383-388. Disponível em:http:// www.scopemed.org/?mno=172340. DOI: https:// doi.org/10.5455/apd.172340

14. Lemos IL, Abreu CN, Sougey EB. Dependência de Internet e de Jogos Eletrônicos: um enfoque cognitivo-comportamental. Rev. psiquiatr. clín. 2014; 41(3):156-167.

15. Conti MA, Jardim AP, Hearst N, Cordás TA,
Tavares H, Abreu CN. Avaliação da equivalência semântica e consistência interna de uma versão em português do Internet Addiction Test (IAT). Revista de Psiquiatria Clínica. [internet] 2012 [acesso em 2018 mai 04] 39:106-110. Disponível em: http://www.scielo.br/scielo. php?pid $=$ S0101-60832012000300007\&script $=$ sci arttext\&tlng=en. DOI: https://doi.org/10.1590/ s0101-60832012000300007.

16. Costa FR. CPS - Escalas de Personalidade de Comrey. 3th ed. São Paulo: Vetor; 2009.112 p.

17. Silva LB, Silva W. As Dimensões da Personalidade: Um olhar sobre tentativas de Compreensão da Personalidade, focando a teoria dos Cinco Grandes Fatores [monografia]. Maceió: Centro Universitário CESMAC; 2018. 18 p.

18. Gervasi AM, Marca LL, Lombardo EMC, Mannino G, Iacolino C, Schimmenti A. Madaptive personality traits and internet addiction symptoms among young adults: a study based on the alternative DSM-5 model for personality disorders. Clinical Neuropsychiatry. 2017;14(1):20-28.

19. Mormizato MS, Ferreira DBB, Souza LSM, Leite RF, Macedo FN, Pimentel D. O uso de internet e Redes Sociais e a Relação com Indícios de Ansiedade e Depressão em Estudantes de Medicina. Ver. Bras. Educ. Med. [internet] 2017 [acesso em 2018 out 03] 41(4):497-504. Disponível em: http://www.scielo.br/scielo. php?pid $=$ S0100-55022017000400497\&script $=$ sci abstract\&tlng=pt. DOI: http://dx.doi. org/10.1590/1981-52712015v41n4rb20160118.

20. American Psychiatric Association. Manual Diagnóstico e Estatístico de Transtornos Mentais. 5. ed. Porto Alegre (RS): Artmed; 2014.

21. Morahan-Martin J, Schumacher P. Loneliness and social uses of the Internet. Computers in Human Behavior. [internet] 2003 [acesso em 2017 mai 13]19(6): 659-671. Disponível em: https://www.sciencedirect.com/science/article/pii/ S0747563203000402?via\%3Dihub. DOI: https://doi. org/10.1016/S0747-5632(03)00040-2

22. Rosa ALM, Arruda EC, Martins ABT. O uso de tecnologias digitais em uma Instituição de Ensino 
Superior: apontamentos sobre a saúde do jovem. Saúde e Pesquisa. [internet] 2016 [acesso em 2019 ago16]9(3):537-545. Disponível em: http:// periodicos.unicesumar.edu.br/index.php/saudpesq/ article/view/5574/2921. DOI: http://dx.doi. org/10.17765/2176-9206.2016v9n3p537-545

Recebido em: 19/02/2019

Aceito em: 22/08/2019 